\title{
Acute necrotising encephalopathy: A rare but important differential diagnosis
}

\author{
M P K Hauptfleisch, ${ }^{1}$ FCPaed (SA), Cert Paed Neuro (SA); L G Scher, ${ }^{2}$ FCPaed (SA); \\ T Pillay, ${ }^{3}$ FCRad Diag (SA), Dip Paed Radiology; T Kilborn, ${ }^{4}$ FRCR (UK)
}

${ }^{1}$ Paediatric Neurology, Department of Paediatrics, Chris Hani Baragwanath Academic Hospital, University of the Witwatersrand, Johannesburg, South Africa

${ }^{2}$ Paediatric Neurology, Department of Paediatrics, Charlotte Maxeke Johannesburg Academic Hospital, University of the Witwatersrand, Johannesburg, South Africa

${ }^{3}$ Department of Radiology, Nelson Mandela Children's Hospital, Honorary Lecturer University of the Witwatersrand, Johannesburg, South Africa ${ }^{4}$ Department of Radiology, Red Cross War Memorial Children's Hospital, University of Cape Town, South Africa

Corresponding author: M P K Hauptfleisch (marc.hauptfleisch@wits.ac.za)

\begin{abstract}
We describe a patient with the classic clinical and radiological findings of acute necrotising encephalopathy and present a review of the current literature. Although it is a rare condition, we believe it should always be considered in an encephalopathic child when the specific radiological findings are seen. This is of particular importance given that early treatment has been reported to result in better outcomes.
\end{abstract}

S Afr J Child Health 2021;15(2):118-120. https://doi.org/10.7196/SAJCH.2021.v15.i2.1812

Acute necrotising encephalopathy (ANE) is a rare cause of acute encephalopathy predominantly occurring in childhood. There have been a few reported cases outside of the East Asian region, where it was first described in 1995 by Mizuguchi et al. ${ }^{[1]}$ The condition typically follows a viral infection, most commonly influenza; however, despite a variety of preceding viral infections being described, the condition is not considered an inflammatory encephalitis. ${ }^{[2]}$ There are no specific laboratory measures to confirm the diagnosis of ANE. Therefore recognition of the characteristic neuroimaging is important to identify cases and allow early treatment.

In this report we give an overview of the neuroimaging findings and clinical presentation to raise awareness of this rare condition that we did not initially consider in our patient.

\section{Case report}

A 3-month-old female patient presented to hospital with an acute onset of abnormal posturing of the right side of her body. Her mother reported that she had a cough and fever the preceding week but no medication was given.

Her birth history was unremarkable, she had been developing appropriately prior to her presentation and there was no relevant family history.

She was a well-grown infant who was encephalopathic and had right-sided extensor dystonia, conjugate eye deviation to the right and clonic movements bilaterally, right more than left. As she was in status epilepticus she was loaded with intravenous phenobarbitone and started on maintenance medication. The seizures stopped but she remained globally dystonic with the right side worse than the left and was noted to have occasional choreiform movements on the right. She remained encephalopathic with her eyes deviated to the right. She was given ceftriaxone and acyclovir.

An urgent computed tomography (CT) scan of the brain performed on admission demonstrated bilateral symmetrical hypodensity of the expanded thalami (Fig. 1).

The differential diagnosis at the time was a possible acute profound hypoxic event, inborn error of metabolism or inflammatory process.

Magnetic resonance imaging (MRI) 1 week later demonstrated predominantly increased signal on T2 involving (A) bilateral thalami and (B) pons (Fig. 2).

Diffusion-weighted imaging (DWI) and apparent diffusion coefficient (ADC) map demonstrated restricted diffusion in the thalami. There were no other abnormalities noted and no abnormal contrast enhancement.

Laboratory investigations did not detect any abnormalities to suggest an inborn error of metabolism or an infective process. The serum lactate was normal and cerebrospinal fluid (CSF) analysis revealed a slightly elevated protein $0.65 \mathrm{~g} / \mathrm{L}$ (normal 0.14 - 0.4 ) with negative viral serology and cultures. There was no pleocytosis, with a lymphocyte count of $2 / \mu \mathrm{L}$. Toxicological studies were normal. There was no evidence of hepatic dysfunction.

The patient had persisting feeding difficulties and dystonia (always worse on the right compared with the left).

The repeat MRI done 8 weeks later shows significant improvement in the thalamic lesions on T2 (Fig. 3) with susceptibility weighting imaging (SWI) demonstrating haemosiderin deposition in both thalami, in keeping with areas of haemorrhage (Fig. 4).

The child was no longer encephalopathic, was able to feed orally but still had residual

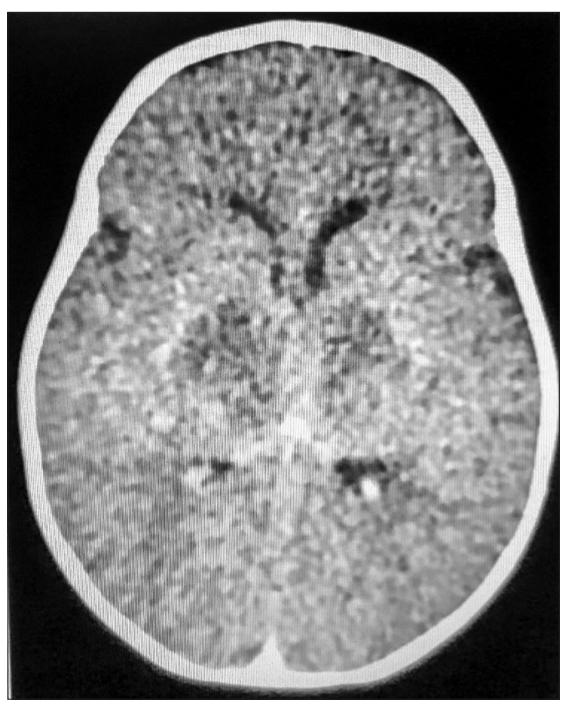

Fig. 1. Contrast computer tomotgraphy of the brain showing bilateral symmetrical thalamic hypodensity with volumetric expansion. 
dystonia (right greater than left) which had improved since presentation.

In view of the above combined clinical findings and reviewing all the neuroimaging a diagnosis of acute necrotising encephalopathy was made.

\section{Discussion}

ANE is a specific type of encephalopathy in otherwise healthy children that follows a rapidly progressive course. Distinctive radiological findings distinguish the condition from other causes of acute encephalopathy, namely bilateral, usually symmetrical T2 hyperintense areas with restricted diffusion in the thalami, posterior putamen, cerebellum and brainstem. ${ }^{[3]}$

ANE has been documented to occur in association with viral infections like influenza $\mathrm{A}$ and $\mathrm{B}$, parainfluenza and human herpesvirus-6 (HHV6). This may imply that the systemic inflammatory responses in young children triggered by viral febrile diseases, as well as the possible modulation thereof with anti-inflammatory drugs, are involved in the pathogenesis of ANE. ${ }^{[2,4]}$ Although the majority of described cases have been in the paediatric age group, with the highest incidence between 6 and 18 months of age, ${ }^{[4]}$ there have been cases documented in adulthood.

Most ANE is sporadic, monophasic and non-recurrent (isolated) but there is a recurrent/familial version - so-called ANE1 related to RANBP2 mutation. In these patients there may be a family history and parents should be warned of the possibility of recurrence. ${ }^{[2]}$ The clinical phenotypes of ANE and ANE1 overlap, with infectious episodes being the trigger for both.

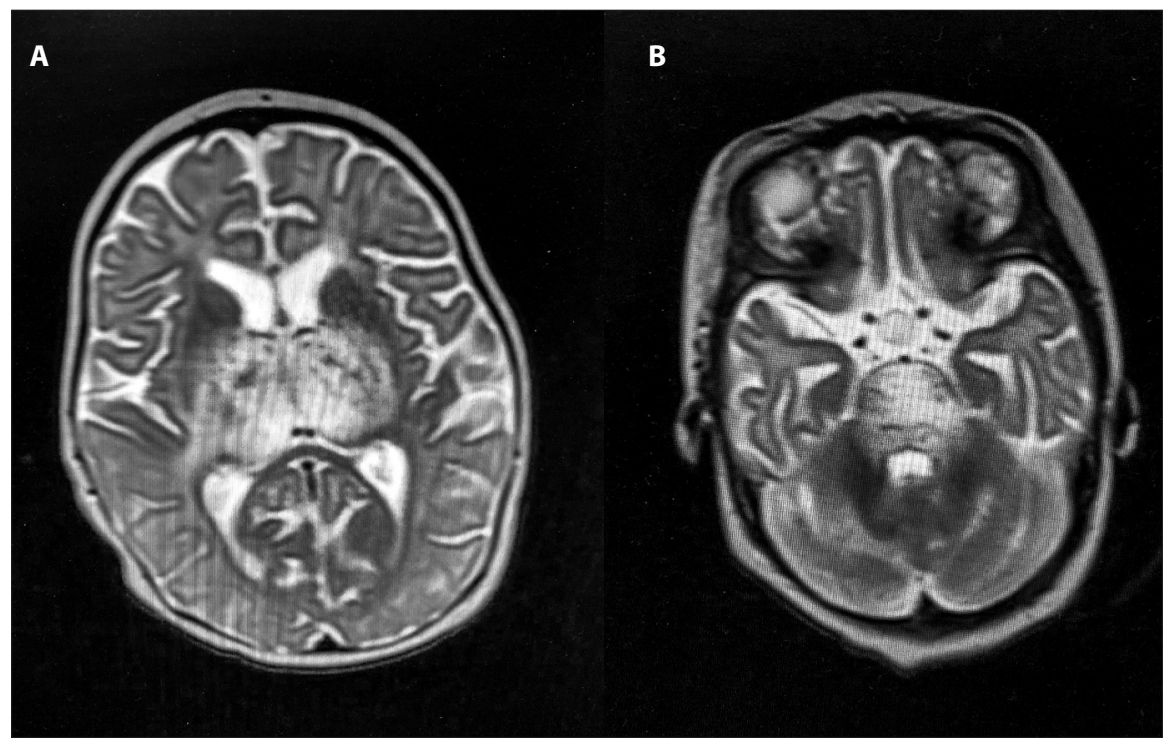

Fig. 2. Magnetic resonance imaging T2 axial showing (A) thalamic hyperintensity and (B) pontine

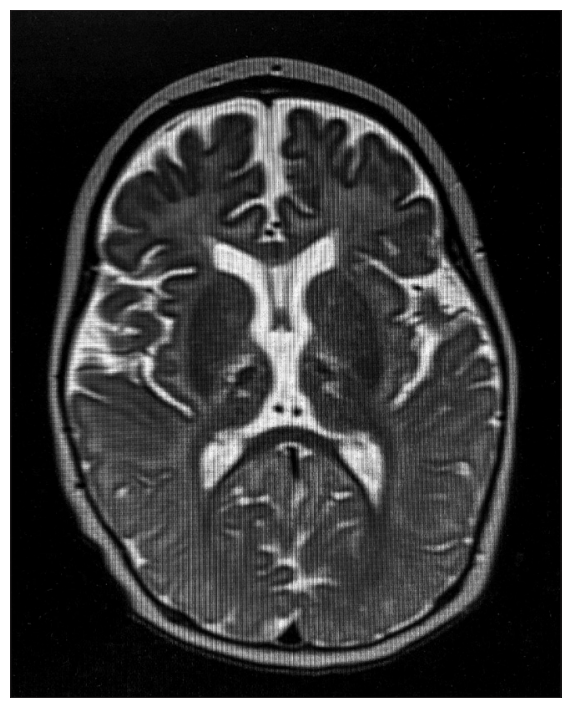

Fig. 3. Magnetic resonance imaging T2 axial showing improvement in thalamic hyperintensity.

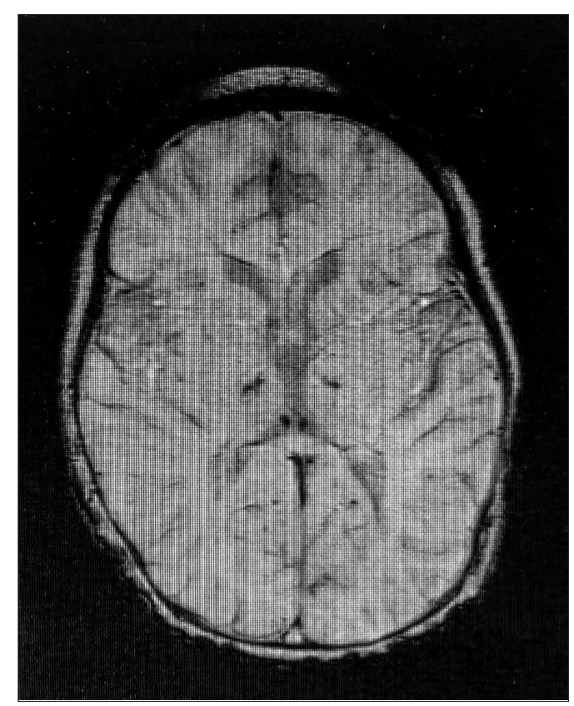

Fig. 4. Susceptibility weighting imaging showing haemosiderin deposition in both thalami.
Clinically ANE resembles many other types of acute encephalopathy. ${ }^{[4]}$ It begins with nonspecific prodromal symptoms: fever, gastrointestinal disturbances, cough and the use of antipyretics in a large portion of patients. ${ }^{[1]}$ This is followed a few days later with seizures, vomiting, dyskinesia, ataxia and altered level of consciousness until reaching stupor and coma usually within 24 hours. In older patients the disease tends to be less severe and may show some asymmetry.

Laboratory findings are neither consistent nor specific. Hepatic dysfunction is seen in more than $75 \%$ of patients; hence the differential of Reye's syndrome, but the transaminitis is transient and there is rarely hyperammonaemia or hypoglycaemia. An increase in CSF protein is seen in $80 \%$ of patients but the degree of elevation is variable. ${ }^{[4]}$

The most important features of ANE are the symmetric brain lesions, which appear at the early stage of coma and show constant pathological features. The lesions are multifocal and involve both the grey and white matter. Unlike Leigh's encephalopathy, ANE shows a fulminant and monophasic course without exception. Acute disseminated encephalomyelits (ADEM) can also present with bilateral symmetrical thalamic abnormalities; however, there are usually other lesions involving the grey and white matter and the lesions typically resolve without cavitation. The neuroradiological changes seen during the course of disease correlate with the pathophysiological changes. There is initial oedema and associated mass effect which slowly resolves; later, petechial haemorrhages are seen and ultimately necrosis. ${ }^{[2]}$ The small petechial haemorrhages are best detected using SWI, as was the case in our patient. The initial oedema causes hypodensities on CT and homogenously prolonged $\mathrm{T} 1$ and $\mathrm{T} 2$ relaxation times on MRI, with restricted diffusion on DWI and ADC mapping. The classic neuroimaging feature described for ANE is a 'target-like' or 'tricolour' appearance of the lesion on MRI ADC mapping; there are three distinct areas of different signal intensity: high signal in the centre, then subsequent lower signal from the cytotoxic oedema, and then once again high signal from the surrounding vasogenic oedema. ${ }^{[5]}$

There is no clear consensus regarding the treatment of ANE but the majority of studies report a benefit of immunomodulatory agents, specifically steroids. However, the timing of treatment, dose and duration vary between institutions. A better outcome has been reported with early treatment. ${ }^{[2]}$ The role of therapeutic hypothermia has been looked into with positive results. ${ }^{[2]}$ 
The prognosis of ANE is poor, with recovery beginning around day 5 and continuing for several weeks. The mortality is as high as $30 \%$ but a good outcome with no neurological sequelae has been reported in around $10 \%$ of cases. ${ }^{[2]}$ An older age of above 4 years at presentation has been associated with a better outcome. Those children who do well show near-complete resolution of the neuroradiological findings, whereas the presence of haemorrhage and necrosis portends to a poorer prognosis. ${ }^{[6]}$ Ongoing research into the condition suggests that ANE can lead to cognitive impairment, attention deficits, fine-motor coordination problems and increased levels of anxiety. ${ }^{[3]}$

\section{Conclusion}

We hope that awareness of this condition will lead to its inclusion in the differential diagnosis of a child presenting with encephalopathy and bilateral thalamic lesions on neuroimaging, especially given that early treatment has been reported to result in better outcomes.

With radiological confirmation of the condition, it is important to warn parents that although it is typically monophasic, there is a possibility of a subsequent attack.

\section{Declaration. None.}

Acknowledgements. Mother of the patient for consenting to the writeup and use of images.
Author contributions. MPKH: write-up and literature review. TK and TP: radiological interpretation and editing. LGS: editing and clinical input. All authors approved the final draft submitted.

Funding. None.

Conflicts of interest. None.

1. Mizuguchi M, Abe J, Mikkaichi K, et al. Acute necrotising encephalopathy of childhood: A new syndrome presenting with multifocal, symmetric brain lesions. J Neurol Neurosurg Psychiatry 1995;58(5):555-561. https://doi. org/10.1136/jnnp.58.5.555

2. Wu X, Wu W, Pan W, Wu L, Liu K, Zhang HL. Acute necrotizing encephalopathy: An underrecognized clinicoradiologic disorder. Mediators Inflamm 2015;2015:792578. https://doi.org/10.1155/2015/792578

3. Williams TA, Brunsdon RK, Burton KLO, et al. Neuropsychological outcomes of childhood acute necrotizing encephalopathy. Brain Dev 2019;41(10):894-900 https://doi.org/10.1016/j.braindev.2019.07.007

4. Mizuguchi M. Acute necrotizing encephalopathy of childhood: A novel form of acute encephalopathy prevalent in Japan and Taiwan. Brain Dev 1997;19(2):8192. https://doi.org/10.1016/s0387-7604(96)00063-0

5. Albayram S, Bilgi Z, Selcuk H, et al. Diffusion-weighted MR imaging findings of acute necrotizing encephalopathy. AJNR Am J Neuroradiol 2004;25(5):792-797.

6. Wong AM, Simon EM, Zimmerman RA, Wang HS, Toh CH, Ng SH. Acute necrotizing encephalopathy of childhood: Correlation of MR findings and clinical outcome. AJNR Am J Neuroradiol 2006;27(9):1919-1923.

Accepted 23 October 2020. 